\title{
Open Science aus Perspektive der Technikfolgenabschätzung
}

\author{
Zu Begriff, gesellschaftlicher Einbettung und \\ möglichen Entwicklungen eines ubiquitären Konzepts
}

\begin{abstract}
Ulrich Riehm, Institut für Technikfolgenabschätzung und Systemanalyse (ITAS), Karlsruher Institut für Technologie (KIT), Karlstr. 11, 76133 Karlsruhe (ulrich.riehm@kit.edu), (1) orcid.org/0000-0002-5107-8305

Michael Nentwich, Institut für Technikfolgen-Abschätzung (ITA) der Österreichischen Akademie der Wissenschaften (ÖAW)

(mnent@oeaw.ac.at), (1) orcid.org/0000-0003-2269-0076
\end{abstract}

Dieser Beitrag nähert sich dem Thema Open Science aus der Perspektive der konzeptionellen Vorbereitung einer umfassenden Technikfolgenabschätzungsstudie. Es werden vier Dimensionen des Konzepts der Offenheit von Wissenschaft unterschieden: freier Zugang, öffentliche Kommunikation, offene Kooperation und die Überwindung gesellschaftlicher Subsysteme. Es wird des Weiteren eine sinnvolle Abgrenzung des Untersuchungsgegenstandes Open Science vorgeschlagen, die bei einer TA-Studie berücksichtigt werden müsste. Nach einer kurzen Darstellung des Status Quo für drei typische Konkretisierungen von Open Science (Open Access, Open-Peer-Review, Wissenschaftsnetzwerke) werden vier Szenarien der zukünftigen Entwicklung von Open Science zur Diskussion gestellt.

\section{Open Science from a technology assessment perspective On the notion, societal embedding, and possible developments of a ubiquitous concept}

This contribution approaches the topic of Open Science from the perspective of preparing and laying the conceptual foundations for a comprehensive technology assessment study. Four dimensions of the concept of openness in science and research are distinguished: open access, public communication, cooperation, and transgression of societal subsystems. Furthermore, a reasonable delimitation of the object of research is proposed, which would form the basis of such a TA study. A short description of the status quo in three typical Open Science arenas (open access, open peer review, and scientific networks) is followed by putting four scenarios of the future development of Open Science up for discussion.

KEYWORDS: open science, open access, citizen science, technology assessment

This is an article distributed under the terms of the Creative Commons Attribution License CCBY 4.0 (https://creativecommons.org/licenses/by/4.0/)

https://doi.org/10.14512/tatup.26.1-2.11

Eingereicht: 07. 04.2017. Angenommen: 06. 06.2017
Die EU-Kommission, ein wichtiger Akteur in der (europäischen) Wissenschaftspolitik, postuliert mit Open Science die große Transformation der Wissenschaft, einen disruptive shiftalles wird anders und alles wird besser:

„Open Science represents a new approach to the scientific process based on cooperative work and new ways of diffusing knowledge by using digital technologies and new collaborative tools. The idea captures a systemic change to the way science and research have been carried out for the last fifty years: shifting from the standard practices of publishing research results in scientific publications towards sharing and using all available knowledge at an earlier stage in the research process." (European Commission 2016, S. 33)

Die Hoffnungen, die die EU-Kommission mit ihrer OpenScience-Politik verbindet, ist, dass Open Science die Wissenschaft glaubwürdiger, zuverlässiger, effizienter und responsiver für die gesellschaftlichen Herausforderungen macht (European Commission 2016, S. 45). Ob überhaupt und in welchem Aus$\mathrm{maß}$ diese große Transformation eintrifft und die Ziele erreicht werden, ist offen. Umfassende, empirisch basierte Studien liegen nicht vor und stehen auch vor erheblichen methodischen Problemen. Und es ist nicht überraschend, dass der disruptive Charakter von Open Science und die damit verbundenen Hoffnungen nicht von allen geteilt und von manchen gar als Bedrohung aufgefasst werden. Als eine wesentliche Gefahr erscheint, dass die auf digitalen Technologien beruhende „Offenheit“ und Transparenz die (wissenschaftsfremde) Kontrolle der Wissenschaft verstärkt und damit wissenschaftliche Autonomie und Freiheit einschränkt (Wilsdon et al. 2017, S. 6; Dickel in diesem Heft).

Der folgende Artikel führt in das Thema „Open Science zwischen Hype und Disruption“ ein. Nach einem kurzen Rückblick auf verwandte Begrifflichkeiten werden vier Dimensionen von „Open“ unterschieden und eine begriffliche Abgrenzung von Open Science vorgeschlagen. Daran anschließend wird auf Open Access, Open-Peer-Review und Wissenschaftsnetz- 
werke als beispielhafte Konkretisierungen von Open Science eingegangen. Angesichts der Komplexität und Weite des Feldes kann dies selbstverständlich nur ein erster, knapper Versuch sein, Parameter zu beschreiben und den möglichen Folgenraum aufzuspannen. Am Schluss des Beitrags stehen vier Szenarien, in welche Richtung sich Open Science entwickeln könnte und das Plädoyer, Open Science einer umfassenden TA zu unterwerfen.

\section{Zur Begriffsgeschichte von Open Science}

Das Open-Science-Konzept bezieht sich auf einige Vorläufer, die allerdings als Reflexionsbegriffe der Wissenschaftsforschung stärker technisch geprägt sind': Der Begriff Cyberscience geht auf einen Artikel von Wouters aus dem Jahr 1996 zurück, den Nentwich (2003, 2005, S. 542-543) in einer umfassenden Studie aufgegriffen hat. Für ihn umfasst Cyberscience die Nutzung vernetzter Computer in der Wissenschaft, und er verbindet damit die Frage, wie Wissenschaft sich dadurch neu organisiert und insgesamt ihren Charakter im Vergleich zur traditionellen Wissenschaft verändert. E-Science hat seine Wurzeln in den frühen 2000er-Jahren und bezieht sich auf große Datensammlungen (was heute unter neuen Vorzeichen auch als Big Data verhandelt wird) und verteilte Computerressourcen, die für den Zugang und den Umgang mit diesen großen Datenmengen benötigt werden (GridComputing). Bei E-Science geht es in erster Linie um eine neue Art vernetzter Computerinfrastruktur (Hey und Trefethen 2002; National e-Science Centre o. J.; Wouters 2006).

Wissenschaftspolitisch motiviert startete die Europäische Kommission mit dem Begriff Science 2.0 eine öffentliche Konsultation. ,2.0“ nimmt dabei auf das sogenannte Web 2.0 und die sich auch im wissenschaftlichen Bereich entwickelnden neuen sozialen Wissenschaftsnetzwerke (Social Media) Bezug. Die Europäische Kommission versteht unter Science 2.0 ,a systemic change in the modus operandi of doing research and organising science“. Dieser Prozess wird nach Auffassung der Kommission durch die digitalen Technologien ermöglicht und durch die Globalisierung der Wissenschaft und die zunehmenden gesellschaftlichen Anforderungen an die Wissenschaft, sich den Grand Challenges zu stellen, befördert (European Commission 2014, S. 1 f.). Dieses Begriffsverständnis ist schon nahe an Open Science. Im Ergebnis der Konsultation übernahm die Kommission dann auch den Begriff Open Science (European Commission 2015, S. 6; Tochtermann 2014).

Kaum von Open Science zu unterscheiden ist der Begriff Digital Science, wie ihn die European Commission (2013) im Kontext des Forschungsprogramms Horizon 2020 verwendet:

„Digital science means a radical transformation of the nature of science and innovation due to the integration of ICT in the research process and the internet culture of openness and sharing. It is more open, more global and collaborative, more creative,

1 Ausführlicher zur Begriffsgeschichte und den Begriffsvarianten bei Nentwich (2003), Jankowski (2007) sowie European Commission (2013). and closer to society. It relies on the use of e-infrastructures, i.e. ICT-based services and tools for data- and computing-intensive research in virtual and collaborative environments. Digital science makes it possible not only to perform research more efficiently but to transform science." (European Commission 2013)

Allerdings ist in der Begriffsbildung auch hier noch das Technische dominant. Mit Open Science ist diese enge technische Fundierung aufgeweicht, wenn auch als wesentliche treibende Kraft für Open Science (und verwandte Konzepte) die digitale und vernetzte Technologieentwicklung anzusehen ist.

\section{Zur gesellschaftlichen Einbettung des Konzepts}

Gleichwohl - das ist in der Technikfolgenabschätzung fast eine Trivialität - braucht die technische Entwicklung, um ihre Wirkungen $\mathrm{zu}$ entfalten, immer eine gesellschaftliche Rahmung und eine sozioökonomische und politische Einbettung (Franzen 2016, S. 279).

Teilweise wird Open Science (und seine Varianten) als „Bewegung“ aus der Wissenschaft charakterisiert, als ,grassroot or bottom-up process" (Tochtermann 2014, S. 1). Natürlich gibt es dieses Moment bei Open Science, insbesondere bei den Pionieren des Internets und den computer- und internetaffinen Communities. Den utopischen Überschuss, den die Entwicklung des Personal Computer und des Internets seit den 1970er-Jahren begleitet hat (Brants 2017), findet man natürlich auch für den Bereich der Wissenschaft. Aber wirkmächtig dürfte Open Science erst dadurch werden - so unsere Vermutung -, dass sich (auch) Wirtschaft und Politik des Themas angenommen und dem mehr oder weniger offenen Konzept ihren Stempel aufgedrückt haben. Dazu zählt, was Taubert und Weingart (2016, S. 12) in Bezug auf den Wandel des wissenschaftlichen Publizierens als Ökonomisierung bezeichnen: Erst auf Grundlage des besonderen Warencharakters wissenschaftlicher Publikationen, der Konzentrationsprozesse im internationalen wissenschaftlichen Verlagswesen und den hohen Gewinnmargen in diesem Bereich konnte sich die umfassende Digitalisierung des wissenschaftlichen $\mathrm{Pu}$ blikationswesens durchsetzen. ${ }^{2}$ Dabei treten die Unternehmen, die traditionell aus dem Publikationsgeschäft kommen, teilweise gar nicht mehr als „Verlage“ auf. Sie suchen vielmehr - ganz im Sinne eines breiten Open-Science-Verständnisses - alle Phasen des wissenschaftlichen Arbeitsprozesses mit Dienstleistungen und „Werkzeugen“ abzudecken und diese möglichst in ein vernetztes System zu integrieren. ${ }^{3}$

Aber auch die Politik setzt Rahmenbedingungen für einen Wandel des wissenschaftlichen (institutionellen) Systems, die gerade in den letzten Jahren mit der Exzellenzinitiative, Elemen-

2 Zu den frühen Anfängen des „elektronischen Publizierens“ Riehm et al. (1992). 3 Beispiele hierfür etwa bei Schonfeld (2017). Der immer noch gemeinhin als Verlag bezeichnete Elsevier "Verlag“ und die Reed Elsevier-Gruppe, zu der Elsevier gehört, bezeichnen sich selbst als "a global provider of information and analytics for professional and business customers across industries" (RELX 2017). 
ten des New Public Management, der Universität als Unternehmen mit Kosten-Leistungs-Rechnung und einer ,,marktvermittelten", leistungsorientierten Mittelvergabe, um nur einige Elemente zu nennen, doch einiges in Bewegung gebracht hat. Wie sich diese neuen Governance-Formen durchsetzen und welche Wirkungen sie zeitigen werden, ist im Einzelnen noch nicht abzusehen (Simon et al. 2016, S. v).

Der Zusammenhang dieser Veränderungen des Wissenschaftssystems mit Open Science müsste erst noch im Detail entwickelt werden. Doch einige Vermutungen können bereits formuliert werden. Die von Maasen und Dickel (2016, S. 226) festgestellte Selbst- und Fremdpolitisierung der Wissenschaft, z. B. durch die freiwillige oder auferlegte Orientierung an normativen Konzepten der Responsivität, Partizipation und Nachhaltigkeit, die wiederum in Citizen Science und Responsible Research und Innovation (RRI) aufgegriffen werden, können auch in ein umfassendes Konzept des Open Science aufgenommen werden (zum Zusammenhang von Open Science, Citizen Science und RRI siehe Vohland und Göbel sowie Häußermann und Heidingsfelder in diesem Heft).

Maasen und Dickel (2016, S. 230) weisen auch auf das Spannungsfeld hin, in dem die heutige Wissenschaft steht: Auf der einen Seite soll sie einem Innovations- und Nützlichkeitsanspruch genügen (welchen Nutzen bringt die Wissenschaft der Gesellschaft) auf der anderen Seite soll sie sich durch „gute wissenschaftliche Praxis" legitimieren. Greift man diesen Gedanken auf, dann verspricht Open Science einen Beitrag zur Bewältigung dieses Spannungsfeldes: Durch eine breitere Verfügbarkeit der wissenschaftlichen Ergebnisse, vor allem aber durch einen größeren gesellschaftlichen Einfluss auf das Was der Wis-

\section{Differenzierungen und Abgrenzungen}

„Offen“ ist eine universell einsetzbare Chiffre, die - positiv konnotiert - gerne einer Vielfalt von Begriffen vorangestellt wird. Bereits 1944 erschien Karl Poppers Buch (auf Englisch) über die „Offene Gesellschaft und ihre Feinde“ ein liberal-demokratisches Manifest gegen Faschismus und Marxismus. George Soros nannte seine 1993 gegründete Stiftung mit Bezug auf Popper „Open Society Institute“ (heute Open Society Foundation). Diese unterstützt auch die Open-Access-Bewegung in der Wissenschaft, u. a. mit der Förderung der Budapest Open Access Initiative im Jahr 2002 sowie der Unterstützung der SPARC (Scholarly Publishing and Academic Resources Coalition) (Open Science Foundation 2014). Was bedeutet aber „offen" bei Open Science? Man kann vier Dimensionen unterscheiden:

- Offenheit der formalen Kommunikation: (Kosten-)freier Zugang, z. B. zu wissenschaftlichen Publikationen oder Forschungsdaten (Hanekop in diesem Heft). Zentral für den Aspekt des offenen Zugangs ist das Konzept des Open Access.

- Offenheit der üblicherweise informellen, vertraulichen Kommunikation: teil-öffentliche Kommunikation, z. B. in Wissenschaftsnetzwerken, bei der Open-Peer-Review und bei der Impactbewertung (inkl. Altmetrics, siehe unten). Öffentliche Kommunikation erzeugt Transparenz, die wissenschaftliches Arbeiten leichter nachvollziehbar, aber auch kontrollierbar macht. „Offene“ öffentliche Kommunikation ist auch mit dem Anspruch verknüpft, dass deren TeilnehmerInnen

\section{Open Science wird durch Wissenschaft und Wissenschafts- organisationen, die IT-Industrie und die multinationalen Wissenschaftsunternehmen forciert.}

senschaft, könnte Open Science die gesellschaftliche Relevanz wissenschaftlicher Ergebnisse fördern. Gleichzeitig würde Open Science die Legitimation der Wissenschaft durch ihren „Kontrollaspekt", der in der größeren Tansparenz mit angelegt ist, erhöhen. Franzen (2016, S. 292) spricht in diesem Zusammenhang von in Open Science angelegten umfassenden Kontrollmechanismen, deren Wirksamkeit das Sozial- und Systemvertrauen in die Wissenschaft wiederherstellen könnte.

Open Science kann als ein technologiegetriebenes, wissenschaftspolitisches Programm charakterisiert werden, das durch Wissenschaft und Wissenschaftsorganisationen, die IT-Industrie und die multinationalen Wissenschaftsunternehmen (Großverlage, Informationsunternehmen, Datenbankanbieter, Internetdienstleister etc.) in Kooperation mit der (europäischen) Wissenschaftspolitik angestoßen und forciert wird (Franzen 2016, S. 279). weder sozialer Diskriminierung unterliegen, etwa nach Geschlecht, Rasse oder Status (Reimann in diesem Heft), noch hinsichtlich der institutionellen Anbindung bzw. geografischen Verortung.

- Offenheit der Wissensproduktion: Zusammenarbeit, wie sie z. B. in der Open-Source-Bewegung, der Wikipedia oder einer „kooperativen Wissenschaft" (CoScience) (Technische Informationsbibliothek (TIB) 2014), auch in Wissenschaftsnetzwerken ihren Ausdruck findet.

- Offenheit des Wissenstransfers: Überwindung der Grenzen gesellschaftlicher Subsysteme, z. B. im Konzept der Citizen Science (Vohland und Göbel in diesem Heft), der „Öffentlichen Wissenschaft“" (Robertson-von Trotha 2012) oder der Open Innovation (European Commission 2016, S. 11; Gerlinger in diesem Heft). 
Wie würde man den Forschungsgegenstand für eine umfassende TA-Studie zu Open Science abgrenzen? Der folgende Vorschlag berücksichtigt die wesentlichen Treiber und Akteure, die zu untersuchenden Bereiche in der Wissenschaft, die mit Open Science verfolgten Ziele sowie die umfassende, disruptive „Vision“ von Open Science:

- Open Science wird befördert durch Attraktivität, breite Verfügbarkeit und die durch Wirtschaft und Politik forcierte Leistungssteigerung vernetzter Informationstechnik. ${ }^{4}$

- Open Science umfasst alle Stadien und Tätigkeiten wissenschaftlicher Arbeit, betrifft alle Artefakte der Wissenschaft und bezieht sich auf alle in der Wissenschaft tätigen und mit dem Wissenschaftssystem verbundenen Akteure.

- Open Science verfolgt unter dem Label „open“ unterschiedliche Ziele: kollaborative wissenschaftliche Arbeit, freien Zugang zu wissenschaftlichen Hervorbringungen, Transparenz der Wissenschaft, Abbau hierarchischer und disziplinärer Grenzen sowie der Grenze zwischen Wissenschaft und Gesellschaft.

- Open Science tritt mit einem weitgehenden Transformationsanspruch für das gesamte wissenschaftliche System an.

\section{Beispielhafte Entwicklungen in Richtung Open Science}

Aus den oben genannten vier Dimensionen werden in der Folge beispielhaft ein paar zentrale Entwicklungen herausgegriffen und überblicksartig beschrieben.

\section{Open Access}

Betrachtet man die Entwicklung der letzten Jahre zu Open Science, dann stand die Forderung nach einem allgemeinen freien Zugang (Open Access) zu Publikationen im Mittelpunkt. Open Access hat sich aber weiter ausdifferenziert und umschließt heute z. B. auch Forschungsdaten, -gegenstände, -methoden und -instrumente (z. B. Software, Modelle, Stoffdatenbanken). Zur parallelen Onlineveröffentlichung von in kostenpflichtigen Zeitschriften publizierten Artikeln in Repositorien durch die jeweiligen Autoren (grüner Weg) tritt in den letzten Jahren verstärkt die Publikation in originären Open-Access-Zeitschriften (goldener Weg) hinzu. Die Finanzierung von originären Open-AccessZeitschriften durch die Autoren bzw. ihre Institutionen über sogenannte APC (article processing charges) ist dabei durchaus umstritten.

Ein früher Kristallisationspunkt der Open-Access-Bewegung war die bereits erwähnte Budapest Open Access Initiative im Jahr 2002. Vorläufer dieser Bewegung findet man aber bis weit in die 1980er-Jahre zurück, als die ersten internetbasierten E-Jour-

4 Der auf konkrete Umsetzung gerichtete Teil des EU-Papiers zu Open Science behandelt beispielsweise ausführlich den Aufbau einer European Open Science Cloud (European Commission 2016, S. 46 ff.). nals und elektronischen Volltextarchive gegründet wurden (Suber 2009). Heute wird von einigen der wissenschaftlichen und politischen Akteure eine vollständige Umstellung des gesamten wissenschaftlichen Publikationssystems (jedenfalls der Zeitschriften) auf (gold) Open Access gefordert, so z. B. im „Amsterdam Call for Action on Open Access“ (o. A. 2016a) oder in der „OA 2020 initiative for the large-scale transition to open access" (o. A. 2016b), und auch für (finanziell) realisierbar gehalten (Schimmer et al. 2015; Crotty 2015; Osborne 2015).

Was weiß man aber nach fast zwanzig Jahren Open-AccessEntwicklung über den „OA-Anteil“ an den wissenschaftlichen Publikationen? Nach dem Open-Science-Monitor (European Commission o. J.) erschienen 2015 elf Prozent der im Web of Science nachgewiesenen Beiträge in originären Open-Access-Zeitschriften (gold) ${ }^{5}$ Ein deutlich höherer Open-Access-Anteil ergibt sich, wenn man self archiving (green), Hybrid-Open-Access und Robin-Hood-Open-Access bzw. rogue OA mit einbezieht und einen maximalen Aufwand für das Auffinden frei zugänglicher Artikel im Internet betreibt. Diesen breiten Ansatz zu Grunde legend, beträgt der Anteil der Artikel, die zwischen 2007 und 2012 publiziert wurden, in der Datenbank Scopus nachgewiesen werden und über das Internet frei zugänglich sind an allen in Scopus nachgewiesenen Artikeln des jeweiligen Publikationsjahrs über $50 \%$ (Archambault et al. 2014, S. ii). Die Autoren führen vier Ursachen an, die zu diesem bedeutenden Wachstum geführt haben: 1) ein generell wachsendes Interesse an Open Access, was zu mehr neuen solcherart publizierten Artikeln führt; 2) gleichzeitig führt dieses gesteigerte Interesse auch dazu, dass mehr bereits publizierte Artikel nachträglich über das Internet zugänglich gemacht werden; 3) zur retrospektiven Veröffentlichung als Open Access tragen auch Open Access policies von wissenschaftlichen Institutionen und Forschungsförderern bei; 4) schließlich ist zu berücksichtigen, dass insgesamt die Zahl der wissenschaftlichen Publikationen ansteigt und deshalb auch die absolute Zahl der OA-Artikel ansteigen wird. Herb (2017) zieht 15 Jahre nach der Budapest Open Access Initiative eine kritische Bilanz: Aktuell würde Open Access weitgehend von den kommerziellen Verlagen angetrieben und die schon Jahre andauernden Konzentrationseffekte im wissenschaftlichen Publikationsmarkt setzten sich ungebrochen fort.

\section{Offene Qualitätssicherung: Altmetrics und Open-Peer-Review}

Ein zweiter wichtiger Bereich für Open Science ist zugleich das Festhalten an und die Weiterentwickeln von Methoden der Qualitätssicherung und Impactbewertung in der Wissenschaft. Die in einigen Disziplinen - nicht in allen - breit etablierte double blind peer review wie auch die Evaluationsindikatoren und quantitativen Metriken (etwa der Impactfaktor) unterliegen einer andauernden Kritik (Franzen 2015; Neidhardt 2016; Wilsdon et al. 2017).

5 ,... proportion of publications from each year in Web of Science (WoS) that are currently available in fully open access journals" (European Commission o. J.). 
Alternative Metriken (altmetrics) gehen über die kommerziellen Datenbanken (Web of Science oder Scopus) hinaus und öffnen die wissenschaftliche Impactbewertung in Richtung nichtwissenschaftlicher Publikationen (z. B. Blogs und Tweets) und anderer, nicht unbedingt wissenschaftlicher Nutzungsformen (z. B. Downloads). Damit könnten sie die herkömmliche, geschlossene Praxis zumindest ergänzen, wenn nicht gar ersetzen. Franzen (2015, S. 228) weist allerdings darauf hin, dass auch die neuen, ,alternativen“ Metriken, die die Nutzung von Publikationen im Internet und neuer Publikationsformen berücksichtigen, als Kontrolltechnologien und als Technologien der Selbstvermarktung (oder des Narzissmus) aufgefasst werden können.

Open-Peer-Review meint die teilweise oder ganz im öffentlichen Diskursraum stattfindende Begutachtung wissenschaftlicher Texte, sei es vor der Veröffentlichung, sei es danach, sei es vollständig, sei es teilweise anonym (Nentwich und König 2010). Auch wenn dieses Konzept schon viel früher erdacht und erprobt wurde als der Begriff der Open Science selbst, ist es doch potenziell ein wichtiges Charakteristikum der letzteren. Bisher hat sich Open-Peer-Review freilich nur in einem sehr begrenzten Umfang durchgesetzt, auch wenn es einige gut funktionierende Beispiele gibt. Der Open-Science-Monitor (European Commission o. J.) gibt den Anteil der Zeitschriften mit Open-Peer-Review auf der Review-Plattform Publons für das Jahr 2016 mit 1,6\% an.

\section{Wissenschaftsnetzwerke}

Der kommunikative und kollaborative Bereich von Open Science scheint dagegen in der wissenschaftlichen Praxis bereits angekommen zu sein (Nentwich und König 2012; Ciber 2016). Dazu gehört nicht nur die Nutzung von Twitter und Facebook durch Wissenschaftler sowie wissenschaftliche Institutionen und ihre Pressestellen (Weingart und Wormer 2016), sondern insbesondere auch die sich in den letzten Jahren mit einigem Erfolg entwickelnden Wissenschaftsnetzwerke vom Typ Mendeley oder ResearchGate. Letzteres hatte im Februar 2016 acht Millionen, Academia.edu über 31 Millionen registrierte Nutzer (Laakso et al. 2017).

Nentwich und König (2012, S. 19 ff.) sehen ein großes Potenzial in den wissenschaftlichen Social-Network-Sites (SNS). Allerdings zweifeln sie, ob diese Angebote schon ihr Alleinstellungsmerkmal gefunden haben, um in der Konkurrenz etwa zur breit einsetzbaren und etablierten E-Mail oder den allgemeinen sozialen Netzwerken vom Typ Facebook bestehen zu können.

Aktuelle Studien deuten darauf hin, dass nur wenige Nutzer in diesen ,academic social network sites“ kommunikativ oder gar kollaborativ aktiv sind (Ciber 2016, S. 9). Ortega (2016) bezeichnet sie als „Diogenes Club“, „where members gather together for common purposes but nonetheless carry out their activities independent of one another". Auffallend ist, dass Wissenschaftsnetzwerke für die (Zweit-)Veröffentlichung eigener Publikationen besonders interessant zu sein scheinen und die Betreiber diese Motivation geschickt aufgreifen, indem sie weitgehend die lästige Arbeit des Einpflegens von Metadaten und des Hochladens von PDFs vorbereiten oder gar abnehmen
(Laakso et al. 2017). Dies könnte dazu führen, dass die Wissenschaftsnetzwerke zu „Technologien des Narzissmus“ werden können - eine Bezeichnung, die Franzen (2015, S. 228) bereits auf alternative Metriken angewendet hat. Ob diese Charakterisierung zum unique selling point der Wissenschaftsnetzwerke werden könnte, bedarf weiterer Beobachtung und Analyse.

\section{Quo vadis Open Science?}

Das wissenschaftliche System entwickelt sich nicht autonom aus sich heraus weiter, sondern steht in spezifischen Spannungsfeldern zur Gesellschaft, die die finanziellen Mittel bereitstellt und Problemlösungen erwartet (Maasen und Dickel 2016). Gleichzeitig eröffnen technologische Entwicklungen neue Potenziale für die wissenschaftliche Arbeit, die eine breite Nutzung finden können, insbesondere wenn ihr ein Nutzenvorteil gegenüber dem herkömmlichen Arbeiten, vielleicht sogar eine visionäre Perspektive zugesprochen wird. Wohin sich Open Science entwickeln wird, ist so keineswegs ausgemacht und unterliegt dem Spiel von Interessen und Ressourcen, über die die Akteure in diesem Spiel verfügen. Weiterhin wird die disziplinenspezifische Heterogenität (Wissenschaftskulturen) eine entscheidende Rolle spielen (siehe Nentwich 2003, S. 107 ff.), ist doch kaum zu erwarten, dass sich sämtliche Fächer und Forschungsgebiete in eine Richtung entwickeln, sondern vielmehr, dass die mit Open Science gegebenen Potenziale in unterschiedlicher Weise genutzt werden.

Wenn man aber darüber spekuliert, was aus Open Science prinzipiell werden könnte, dann bieten sich die folgenden Alternativen an:

- Disruptiver Utopismus - Alles wird anders: Darunter ist zu verstehen, was gegenwärtig die Europäische Kommission programmatisch vertritt. Solche Programme könnten zwar eine mobilisierende gesellschaftliche Wirkung entfalten, rufen aber auch Gegenkräfte hervor, die darin keine Befreiung, sondern eine Bedrohung sehen.

- Open Science als Mittel gesteigerter Kontrolle und Konkurrenz: Eine mögliche Entwicklung wäre auch, dass das, was durch Transparenz und Effizienzzuwachs bei Open Science gewonnen wird, in eine Verschärfung der innerwissenschaftlichen Konkurrenz mündet. Kommerzielle Interessen, etwa aus dem Patent- und Lizenzgeschäft, wären hiermit kompatibel: „Open Science, however, does not mean free science. It is essential to ensure that intellectual property is protected before making knowledge publicly available" (European Commission 2016, S. 42).

- Ein neuer Typ Wissenschaft: Open Science könnte sich aber auch zu einem neuen Typus von Wissenschaft entwickeln, der die herkömmliche Wissenschaft zwar nicht unbeeinflusst lässt, aber auch nicht disruptiv verändert. Open Science könnte als eine Art Dach für Konzepte fungieren, die sich in den letzten Jahren auch aus dem disziplinären wissenschaftlichen System herausgebildet haben. $\mathrm{Zu}$ nennen sind 
hier nicht nur Citizen Science und RRI, sondern auch transdisziplinäre und transformative Forschung. ${ }^{6}$

- Das Ende der professionalisierten Wissenschaft: Durch die Öffnung der Wissenschaft für gesellschaftliche Gruppen und Anliegen sieht Dickel (in diesem Heft) das Ende einer selbstgesteuerten und auf professionellen Standards und Rollen beruhenden Wissenschaft. Was dann an ihre Stelle treten könnte, bleibt allerdings offen.

Mit anderen Worten: Open Science erschließt ein weites Feld für die Technikfolgenabschätzung, das die Beiträge zu diesem Themenschwerpunkt nur in Ansätzen abdecken können. Eine breit angelegte TA-Studie (im Sinne einer comprehensive TA) zu dieser offensichtlich wirkmächtigen, potenziell disruptiven Entwicklung durchzuführen, wäre lohnend. Erst die Zusammenführung unterschiedlicher (disziplinärer) Perspektiven anstelle von mehr oder weniger disziplinären Einzelbetrachtungen und die systematische Anwendung bewährter TA-Methoden, insbesondere von Szenario-Techniken unter Einbeziehung der unterschiedlichen Akteure, wäre in der Lage, robuste Antworten auf die vielen offenen Fragen zu geben, die Chancen und Risiken abzuschätzen sowie optionale Handlungsstrategien zu entwickeln.

\section{Danksagung}

Die Autoren danken den beiden Gutachtern für wertvolle Verbesserungsvorschläge im Begutachtungsverfahren.

\section{Literatur}

Archambault, Éric et al. (2014): Proportion of Open Access Papers Published in Peer-Reviewed Journals at the European and World Levels, 1996-2013. RTD-B6-PP-2011-2: Study to Develop a Set of Indicators to Measure Open Access. Science-Metrix. Montreal (D 1.8 version: 11 p). Online verfügbar unter http://digitalcommons.unl.edu/cgi/viewcontent.cgi?article=1007\& context=scholcom, zuletzt geprüft am 08.06.2017.

Brants, Dietrich (2017): FlowerPowerDatenTerror. Wie die Gegenkultur die Cyberkultur prägt (SWR2 Essay). Online verfügbar unter http://www.swr.de/ swr2/programm/sendungen/essay/swr2-essay-flowerpowerdatenterror/-/ id=659852/did=18984524/nid=659852/1cbfavu, zuletzt geprüft am 08. 06. 2017.

Ciber (2016): Early Career Researchers: The Harbingers of Change? Year One (2016). Final Report from CIBER. Online verfügbar unter http://publishingresearchconsortium.com/index.php/prc-documents/ prc-research-projects/58-ecrs-year-one-2016-full-report/file, zuletzt geprüft am 08. 06.2017.

Crotty, David (2015): The Global Gold Open Access "Flip": A Realistic Plan or Magical Thinking? (The Scholarly Kitchen Blog). Online verfügbar unter https:// scholarlykitchen.sspnet.org/2015/09/24/the-global-gold-open-access-flip-arealistic-plan-or-magical-thinking, zuletzt geprüft am 08.06.2017.

European Commission (o. J.): Open Science Monitor. Open Access to publications. Online verfügbar unter https://ec.europa.eu/research/openscience/index. cfm?section=monitor\&pg=access, zuletzt geprüft am 08. 06.2017.

6 In dem von Wilsdon et al. (2017, S. 6) vorgelegten Papier für die Europäische Kommission wird interessanterweise als eine Barriere für Open Science die Forschung in disziplinären Strukturen angegeben, die es zu überwinden gelte.
European Commission (2013): Digital Science in Horizon 2020. Concept Paper of the Digital Science Vision, and its Integration in the Horizon 2020 Programme. Online verfügbar unter http://ec.europa.eu/information_society/newsroom/ cf/dae/document.cfm?doc_id=2124, zuletzt geprüft am 08.06.2017.

European Commission (2014): 'Science 2.0': Science in Transition. Public Consultation - Background document. Online verfügbar unter https://ec.europa.eu/research/consultations/science-2.0/background.pdf, zuletzt geprüft am 08. 06.2017.

European Commission (2015): Validation of the Results of the Public Consultation on Science 2.0: Science in Transition. Online verfügbar unter https://ec.europa.eu/research/consultations/science-2.0/science_2_0_final_ report.pdf, zuletzt geprüft am 08. 06.2017.

European Commission (2016): Open Innovation, Open Science, Open to the World. A Vision for Europe. Brüssel: European Commission.

Franzen, Martina (2015): Der Impact Faktor war gestern. Altmetrics und die Zukunft der Wissenschaft. In: Soziale Welt 66 (2), S. 225-242.

Franzen, Martina (2016): Open Science als wissenschaftspolitische Problemlösungsformel? In: Dagmar Simon, Andreas Knie, Stefan Hornbostel und Karin Zimmermann (Hg.): Handbuch Wissenschaftspolitik. 2. Aufl. 2016. Wiesbaden: Springer Fachmedien, S. 279-296. DOI: 10.1007/978-3-658-05455-7_23.

Herb, Ulrich (2017): Open Access zwischen Revolution und Goldesel. Eine Bilanz fünfzehn Jahre nach der Erklärung der Budapest Open Access Initiative. In: Information, Wissenschaft \& Praxis 68 (1), S. 1-10.

Hey, Tony; Trefethen, Anne E. (2002): The UK e-Science Core Programme and the Grid. In: Future Generation Computer Systems 18 (8), S. 1017-1031. Online verfügbar unter https://eprints.soton.ac.uk/257644/1/ UKeScienceCoreProg.pdf, zuletzt geprüft am 09. 06.2017.

Jankowski, Nicholas W. (2007): Exploring e-Science: An Introduction. In: Journal of Computer-Mediated Communication 12 (2), S. 549-562. DOI: 10.1111/j.10836101.2007.00337.x.

Laakso, Michael; Lindman, Joho; Shen, Cenyu; Nyman, Linus; Björk, Bo-Christer (2017): Research Output Availability on Academic Social Networks: Implications for Stakeholders in Academic Publishing. In: Electronic Markets. DOI: 10.1007/s12525-016-0242-1.

Maasen, Sabine; Dickel, Sascha (2016): Partizipation, Responsivität, Nachhaltigkeit. Zur Realfiktion eines neuen Gesellschaftsvertrags. In: Dagmar Simon, Andreas Knie, Stefan Hornbostel und Karin Zimmermann (Hg.): Handbuch Wissenschaftspolitik. 2. Aufl. 2016. Wiesbaden: Springer Fachmedien, S. 225-242. DOI: 10.1007/978-3-658-05455-7_21.

National e-Science Centre (о. J.): Defining e-Science. Online verfügbar unter http://www.nesc.ac.uk/nesc/define.html, zuletzt geprüft am 08.06.2017. Neidhardt, Friedhelm (2016): Selbststeuerung der Wissenschaft durch PeerReview-Verfahren. In: Dagmar Simon, Andreas Knie, Stefan Hornbostel und Karin Zimmermann (Hg.): Handbuch Wissenschaftspolitik. 2. Aufl. 2016. Wiesbaden: Springer Fachmedien Wiesbaden (Springer Reference Sozialwissenschaften), S. 261-277. DOI: 10.1007/978-3-658-05455-7_22.

Nentwich, Michael (2003): Cyberscience. Research in the Age of the Internet. Wien: Austrian Academy of Sciences Press.

Nentwich, Michael (2005): Cyberscience: Modelling ICT-induced changes of the scholarly communication system. In: Information, Communication \& Society 8 (4), S. 542-560. DOI: 10.1080/13691180500418451.

Nentwich, Michael; König, René (2010): Peer Review 2.0: Herausforderungen und Chancen der wissenschaftlichen Qualitätskontrolle im Zeitalter der Cyber-Wissenschaft. In: Martin Gasteiner und Peter Haber (Hg.): Digitale 
Arbeitstechniken für die Geistes- und Kulturwissenschaften. Wien: Böhlau UTB, S. 143-163.

Nentwich, Michael; König, René (2012): Cyberscience 2.0. Research in the Age of Digital Social Networks. Frankfurt am Main: Campus (Interaktiva, Schriftenreihe des Zentrums für Medien und Interaktivität, Gießen, 11).

o. A. (2016a): Amsterdam Call for Action on Open Science. Amsterdam. Online verfügbar unter https://english.eu2016.nl/binaries/eu2016-en/documents/ reports/2016/04/04/amsterdam-call-for-action-on-open-science/ amsterdam-call-for-action-on-open-science.pdf, zuletzt geprüft am 08. 06.2017.

O. A. (2016 b): OA2020 Mission. Expression of Interest in the Large-scale Implementation of Open Access to Scholarly Journals. Online verfügbar unter https://oa2020.org/mission, zuletzt geprüft am 08. 06.2017.

Open Science Foundation (2014): What Is "Open Access"? Online verfügbar unter https://www.opensocietyfoundations.org/explainers/what-open-access, zuletzt geprüft am 08.06.2017.

Ortega, José Luis (2016): Academic Social Networks: Collaborative Environments or Diogenes Clubs? (The Scholarly Kitchen Blog). Online verfügbar unter https://scholarlykitchen.sspnet.org/2016/12/08/guest-post-jose-luis-ortegaacademic-social-networks-collaborative-environments-or-diogenes-clubs, zuletzt geprüft am 08.06.2017.

Osborne, Robin (2015): A Simple but Flawed Case for the Financial Viability of Open Access. Review of Schimmer et al. (2015) Disrupting the Subscription Journals' Business Model for the Necessary Large-scale Transformation to Open Access: A Max Planck Digital Library Open Access Policy White Paper. Online verfügbar unter https://www.scienceopen.com/document/ reviews?vid=05762b81-434d-407e-b33c-1b6cccf2f128, zuletzt geprüft am 08. 06.2017.

RELX (2017): About Us. Online verfügbar unter http://www.relx.com/AboutUs, zuletzt geprüft am 07.04.2017.

Riehm, Ulrich; Böhle, Knud; Gabel-Becker, Ingrid; Wingert, Bernd (1992): Elektronisches Publizieren. Eine kritische Bestandsaufnahme. Berlin: Springer.

Robertson-von Trotha, Caroline Y. (2012): Öffentliche Wissenschaft im Spiegel der Web 2.0-Kultur. In: Caroline Y. Robertson-von Trotha und Jesus Munoz Morcillo (Hg.): Öffentliche Wissenschaft und Neue Medien. Die Rolle der Web 2.0-Kultur in der Wissenschaftsvermittlung. Karlsruhe: KIT Scientific Publishing, S. 19-35.

Schimmer, Ralf; Geschuhn, Kai Karin; Vogler, Andreas (2015): Disrupting the Subscription Journals' Business Model for the Necessary Large-scale Transformation to Open Access. A Max Planck Digital Library Open Access Policy White Paper (ScienceOpen Research). Online verfügbar unter https://www.scienceopen.com/document?vid=05762b81-434d-407e-b33c1b6cccf2f128, zuletzt geprüft am 08. 06.2017.

Schonfeld, Roger C. (2017): When is a Publisher Not a Publisher? Cobbling Together the Pieces to Build a Workflow Business (The Scholarly Kitchen Blog). Online verfügbar unter https://scholarlykitchen.sspnet. org/2017/02/09/cobbling-together-workflow-businesses, zuletzt geprüft am 08. 06.2017.

Simon, Dagmar; Knie, Andreas; Hornbostel, Stefan; Zimmermann, Karin (Hg.) (2016): Handbuch Wissenschaftspolitik. 2. Aufl. 2016. Wiesbaden: Springer Fachmedien Wiesbaden (Springer Reference Sozialwissenschaften). DOI: 10.1007/978-3-658-05455-7.

Suber, Peter (2009): Timeline of Open Access Movement. Formerly Called the Timeline of the Free Online Scholarschip Movement. Online verfügbar unter

http://legacy.earlham.edu/ peters/fos/timeline.htm, zuletzt aktualisiert am 09. 02.2009, zuletzt geprüft am 26.06.2017.

Taubert, Niels; Weingart, Peter (2016): Wandel des wissenschaftlichen Publizierens - eine Heuristik zur Analyse rezenter Wandlungsprozesse. In: Peter Weingart und Niels Taubert (Hg.): Wissenschaftliches Publizieren. Zwischen Digitalisierung, Leistungsmessung, Ökonomisierung und medialer Beobachtung. Berlin: De Gruyter (Interdisziplinäre Arbeitsgruppen Forschungsberichte, 38), S. 3-38. Online verfügbar unter https://www.degruyter.com/downloadpdf/books/9783110448115/ 9783110448115-001/9783110448115-001.pdf, zuletzt geprüft am 08. 06.2017.

Technische Informationsbibliothek (TIB) (2014): CoScience - gemeinsam forschen und publizieren im Netz. Version 1.0. Technische Informationsbibliothek (TIB). Hannover.

Tochtermann, Klaus (2014): Comments on 'Science 2.0'. Response to the European Commission Consultation on 'Science in Transition'. Online verfügbar unter http://ec.europa.eu/research/consultations/science-2.0/science_2.0_ position_papers.zip, zuletzt geprüft am 08. 06.2017.

Weingart, Peter; Wormer, Holger (2016): Wissenschaftskommunikation als demokratisches Grundprinzip. In: Technikfolgenabschätzung - Theorie und Praxis (TATUP) 25 (1), S. 8-16. Online verfügbar unter http://www.tatupjournal.de/tatup161_wew016a.php, zuletzt geprüft am 08.06.2017.

Wilsdon, James; Bar-Ilan, Judit; Frodeman, Robert; Lex, Elisabeth; Peters, Isabella; Wouters, Paul (2017): Next-generation Metrics: Responsible Metrics and Evaluation for Open Science. Report of the European Commission Expert Group on Altmetrics. European Commission. Brüssel. Online verfügbar unter https://ec.europa.eu/research/openscience/pdf/report.pdf, zuletzt geprüft am 08. 06.2017.

Wouters, Paul (2006): What is the Matter with e-Science? Thinking Aloud about Informatisation in Knowledge Creation. In: Pantaneto Forum (23), S. 1-9.
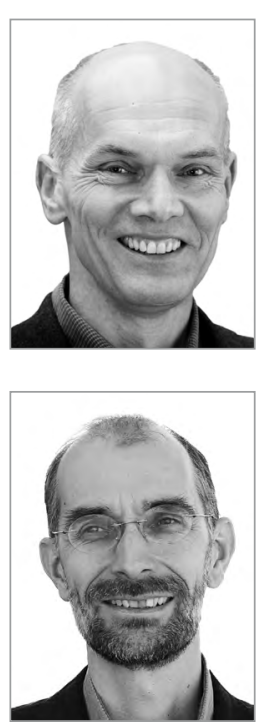

\section{ULRICH RIEHM}

ist Diplom-Soziologe und arbeitet als wissenschaftlicher Mitarbeiter des Instituts für Technikfolgenabschätzung und Systemanalyse (ITAS) des Karlsruher Instituts für Technologie. Er beschäftigt sich in seiner Forschung seit den 1980er-Jahren mit den Veränderungen im wissenschaftlichen Publizieren und der Buchbranche.

\section{PD DR. MICHAEL NENTWICH}

ist promovierter Jurist, habilitierter Wissenschaftsund Technikforscher sowie Direktor des Instituts für Technikfolgen-Abschätzung (ITA) der Österreichischen Akademie der Wissenschaften in Wien (seit 2006). Autor u. a. von Cyberscience (2003) und Cyberscience 2.0 (2012). 